\title{
An Update on Surgical and Medical Management Strategies for Intracerebral Hemorrhage
}

\author{
Natalie Kreitzer, $\mathrm{MD}^{1,2}$ Opeolu Adeoye, MD, MS $\mathrm{MS}^{1,2,3}$ \\ 1 University of Cincinnati Neuroscience Institute, Cincinnati, Ohio \\ ${ }^{2}$ Department of Emergency Medicine, University of Cincinnati, \\ Cincinnati, Ohio \\ ${ }^{3}$ Department of Neurosurgery, University of Cincinnati, \\ Cincinnati, Ohio

\begin{abstract}
Address for correspondence Opeolu Adeoye, MD, MS, Division of Neurocritical Care, Department of Emergency Medicine, University of Cincinnati, College of Medicine, 231 Albert Sabin Way, Cincinnati, Ohio 45267-0769 (e-mail: Opeolu.Adeoye@uc.edu).
\end{abstract}

Semin Neurol 2013;33:462-467.

\begin{abstract}
Keywords

- intracerebral hemorrhage

- surgical management

- medical management

Intracerebral hemorrhage (ICH) accounts for 10 to $15 \%$ of all strokes and is one of the major causes of stroke-related death and disability. After the initial hemorrhage, further bleeding and edema contribute to secondary damage and worsened outcomes. As such, goals of previous and ongoing trials are to prevent continued bleeding, as well as mitigate the impact of cerebral edema. Although no trials have shown a definite functional outcome benefit with a given intervention, much progress has been made recently. This review focuses on recent developments that inform the acute management of $\mathrm{ICH}$.
\end{abstract}

Intracerebral hemorrhage (ICH) is an important cause of stroke-related death and disability. Intracerebral hemorrhage accounts for $\sim 10$ to $15 \%$ of all strokes in the United States with an incidence of 12 to 15 cases per 100,000 per year. ${ }^{1,2}$ The incidence is higher in Asian countries, at $~ 20$ to $30 \%$ of all strokes. ${ }^{3}$ Mortality is almost $50 \%$ at 30 days, and half of these deaths occur within the first 24 hours. ${ }^{1}$ Although no intervention tested in clinical trials to date has significantly demonstrated improved functional outcome following $\mathrm{ICH}$, recently completed and ongoing trials may have a direct impact on the acute care of ICH patients. In this review, we discuss acute management of ICH in light of recently completed and ongoing ICH clinical trials.

\section{Prognosis in Intracerebral Hemorrhage}

An important prognostic factor in $\mathrm{ICH}$ is the presenting hematoma volume and subsequent hematoma expansion. Hematoma expansion occurs in upwards of $40 \%$ of ICH patients, ${ }^{4,5}$ typically occurs within the first few hours, and portends neurologic deterioration, poorer functional outcome, and increased mortality. Each $10 \%$ increase in hematoma size from baseline has been associated with a $5 \%$ increase in mortality and $16 \%$ chance of worse functional outcome. ${ }^{6}$ Unfortunately, trials of hemostatic agents for ICH (discussed in more detail below) have not shown improved functional outcomes. $^{7,8}$

Concurrent intraventricular hemorrhage (IVH) is another factor that has been associated with worse outcome after ICH. Intracerebral hemorrhage may lead to obstructive hydrocephalus causing acute intracranial hypertension that may result in herniation syndromes. Mortality rates of 50 to $80 \%$ have been reported in ICH patients with IVH. ${ }^{9,10}$ Emergent placement of an extraventricular drain (EVD) may be lifesaving and recent studies suggest administration of recombinant tissue plasminogen activator (rt-PA) or urokinase may facilitate resolution of the IVH. ${ }^{11,12}$ A phase III clinical trial is ongoing to determine if resolution of IVH with rt-PA is associated with improved long-term clinical outcome.

Other factors that have been associated with poorer outcome after ICH include age, infratentorial ICH location, edema, and warfarin or other anticoagulant use. A variety of prognostic tools have been published for $\mathrm{ICH}^{10,13}$ However, clinicians must be cautious regarding the so-called self-fulfilling prophecy of ICH management, ${ }^{14}$ whereby withdrawal of care or initiation of comfort care measures early in the clinical course has become the leading proximate cause of death after ICH. This is particularly important given recent data suggesting that functional outcomes after ICH continue
Issue Theme Advanced Cerebrovascular Disease Management; Guest Editor, Jason Mackey, MD, MS
Copyright @ 2013 by Thieme Medical Publishers, Inc., 333 Seventh Avenue, New York, NY 10001, USA. Tel: +1(212) 584-4662.
DOI http://dx.doi.org/ 10.1055/s-0033-1364210. ISSN 0271-8235. 
improve past the 6-month mark and reports of early morbidity and mortality may misrepresent potential outcomes. ${ }^{15}$

\section{Acute Management of Intracerebral Hemorrhage}

Intracerebral hemorrhage is an emergency. The acute clinical presentation depends on the location of the lesion within the brain. A sudden focal neurologic deficit, change in consciousness, headache, or vomiting are common presentations. A computed tomography (CT) scan or magnetic resonance imaging (MRI) is required to confirm a diagnosis of ICH. Care should always begin with airway stabilization, breathing, and circulation (ABCs). Specifically, many patients require active airway management with endotracheal intubation and mechanical ventilation secondary to their depressed mental status. The head of the bed should be elevated to 30 degrees at all times, and the neck kept in a midline position. Adequate analgesia and sedation should be provided. Other specific measures such as blood pressure management are crucial as well. Acute emergency department (ED) interventions that can minimize bleeding are critical for improved outcomes. These are discussed further below and in addition to the ABCs include timely diagnosis, blood pressure management, and reversal of anticoagulation.

\section{Imaging}

Initial imaging and diagnosis of ICH is almost always made on a noncontrast head CT. Addition of CT angiography (CTA) may reveal a "spot sign," a hyperdensity within the hematoma corresponding to contrast extravasation, which has been associated with subsequent hematoma expansion. ${ }^{16-18}$ In the largest prospective observational study published to date, 30\% of 268 ICH patients with ICH volume less than $100 \mathrm{~mL}$ who underwent CTA within 6 hours of symptom onset had a spot sign. ${ }^{18}$ Of these, $61 \%$ experienced hematoma expansion compared with $22 \%$ of patients who had no spot sign. Sensitivity of the spot sign for predicting hematoma expansion was $51 \%$ and specificity was $85 \%{ }^{18}$

Early CTA may also reveal secondary causes of ICH such as arteriovenous malformations and aneurysms. Magnetic resonance imaging (MRI), MR angiography (MRA), catheter angiography also play a significant role in identifying secondary causes of ICH and may be essential prior to enrolling patients in clinical trials testing administration of rt-PA for hastening clot resolution. The most recent American Stroke Association (ASA) guidelines do not specify which ICH patients may benefit from vascular imaging for evaluation of secondary causes. ${ }^{1}$ However, recent clinical experience suggests such imaging may be warranted in more patients than currently recognized, particularly in the young (-Fig. 1).

\section{Blood Pressure Control}

Effective management of blood pressure, especially in the acute phase of ICH, is imperative.

The European Stroke Initiative (EUSI) in 2006 recommended that antihypertensive treatment be initiated if systolic blood pressure (SBP) is $\geq 180 \mathrm{~mm} \mathrm{Hg},{ }^{19}$ which is similar to the ASA guidelines from 1999, which recommended treatment for SBP $\geq 180 \mathrm{~mm} \mathrm{Hg}$ or diastolic blood pressure (DBP) $\geq 105 \mathrm{~mm} \mathrm{Hg} .{ }^{20}$ The 2007 ASA guidelines recommend treatment if SBP is $\geq 180 \mathrm{~mm} \mathrm{Hg}$ or mean arterial pressure (MAP) is $\geq 130 \mathrm{~mm} \mathrm{Hg}$ and there is no evidence or suspicion of elevated ICP. If there is concern for elevated ICP, then patients should have intracranial pressure (ICP) monitoring, and cerebral perfusion pressure (CPP) should be maintained between 60 to $80 \mathrm{~mm} \mathrm{Hg.}{ }^{21}$ In 2010, the ASA guidelines were further modified and recommended a goal SBP $<160 \mathrm{~mm} \mathrm{Hg}$ or a MAP below $110 \mathrm{~mm} \mathrm{Hg.}{ }^{1}$ The guidelines further state that if SBP $>180$, or MAP $>130$ and increased ICP is suspected, then ICP monitoring is recommended. Intravenous, short-acting medications are recommended for treating acute hypertension in ICH. Recommended medications include labetalol (5-20 mg every 15 minutes or infusion of $2 \mathrm{mg} / \mathrm{min}$ ), nicardipine (infusion of 5-15 mg/h), esmolol (bolus $250 \mu \mathrm{g} / \mathrm{kg}$, infusion 25-300 $\mu \mathrm{g} /$ $\mathrm{kg} / \mathrm{min}$ ), enalaprilat (1.25-5 mg every 6 hours with starting dose of $0.625 \mathrm{mg}$ intravenously [IV]), hydralazine (5-10 mg every 30 minutes, or infusion of $1.5-5 \mu \mathrm{g} / \mathrm{kg} / \mathrm{min}$ ), nitroprusside (infusion $0.1-10 \mu \mathrm{g} / \mathrm{kg}$ ), or nitroglycerin (infusion 20-400 $\mu \mathrm{g} / \mathrm{min}$ ). In 2010, a goal SBP of $140 \mathrm{~mm} \mathrm{Hg}$ was considered "probably safe," which was a modification from the 2007 guidelines. The evolution of these practice guidelines reflects the growing evidence for blood pressure management in ICH.

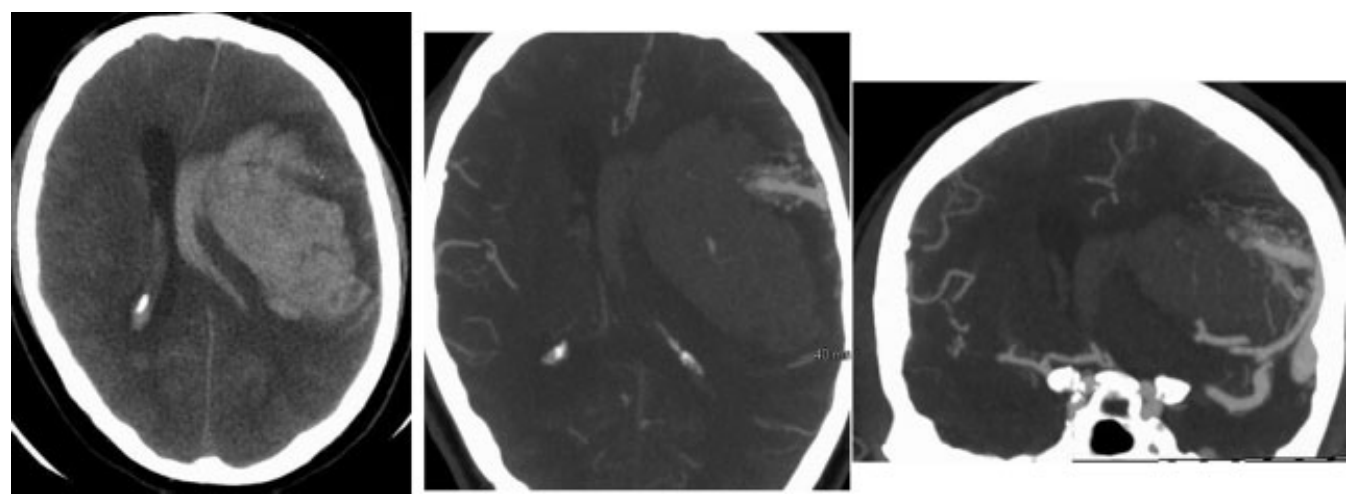

Fig. 1 A 43-year-old woman with poorly controlled diabetes and hypertension who presented with an intracerebral hemorrhage. A dural arteriovenous fistula is demonstrated on computed tomography angiography. 
The pilot Intensive Blood Pressure Reduction in Acute Cerebral Hemorrhage Trial (INTERACT) trial evaluated 404 patients randomized to early BP lowering (systolic target $140 \mathrm{~mm} \mathrm{Hg}$ ) versus standard management (target $<180$ $\mathrm{mm} \mathrm{Hg}$ ) within 6 hours of onset. ${ }^{22}$ Rapid blood pressure reduction following ICH was found to be safe in INTERACT. Further, the intensive blood pressure control group demonstrated reduced hematoma expansion (14\% vs. $36 \%$ ), but these results were not statistically significant. ${ }^{22}$ Subsequently, ATACH and INTERACT2 were conducted and ATACH-2 is ongoing. Given the current available evidence, we believe a target SBP of $140 \mathrm{~mm} \mathrm{Hg}$ may be safe in the early clinical ( $<6$ hours) course of acute ICH. It remains unclear whether the blood pressure may be safely dropped to $140 \mathrm{~mm} \mathrm{Hg}$ in the ultraearly period ( $<3.5$ hours) and whether such early aggressive blood pressure control would result in improved outcomes.

\section{Hemostatics}

Various hemostatic agents have been and are currently being tested as therapies for the management of ICH. One hemostatic agent of high interest is recombinant activated factor VII ( $r F V I I a)$. rFVIIa has been proven to significantly reduce hematoma expansion in phase IIb $(n=339)^{7}$ and phase III $(n=841)^{8}$ trials when given within 4 hours of symptom onset. Unfortunately, clinical efficacy in terms of improved outcomes was not demonstrated in those trials. A proposed explanation for this lack of efficacy for improving outcomes despite reducing hematoma expansion was the inclusion of an unselected patient population. Particularly, rFVIIa treatment was associated with an $\sim 20 \%$ absolute increase in ischemic strokes and myocardial infarction in the phase III trial. ${ }^{23}$ Thus, exposure of patients who were not bound to experience hematoma expansion to this risk may have blunted any benefits from reducing hematoma expansion in patients who experienced expansion. ${ }^{8}$ Patients with large hematomas were also included although they were bound to have a poor outcome regardless of hematoma expansion. As such, it has been suggested that patients aged 70 years or younger, ICH volume $<60 \mathrm{~mL}$ with minimal IVH and treated within 2.5 hours may be a subset of patients who would benefit from rFVIIa. ${ }^{24}$ Patient selection based on the spot sign may also select those patients most likely to experience hematoma expansion.

Two ongoing trials are using early CTA to select ICH patients for treatment with rFVIIa. The Spot Sign for Predicting \& Treating ICH Growth (STOP-IT) study is an ongoing phase II, randomized, multicenter, double-blind, placebocontrolled trial with the primary goals of (1) determining the sensitivity and specificity of the CTA spot sign for predicting hematoma expansion, (2) determining the feasibility of using CTA to identify ICH patients at high risk of hematoma expansion and to select spot-positive patients for randomization to treatment with rFVIIa or placebo, and (3) determining the rate of hematoma expansion among spot-positive patients at 24 hours. The planned enrollment is for 184 total patients. ${ }^{25}$ The Spot Sign Selection of Intracerebral Hemorrhage to Guide Hemostatic Therapy (SPOTLIGHT) study is also an ongoing, phase II, randomized, multicenter, double-blind, placebo-controlled trial with similar goals of randomizing spot-positive ICH patients to treatment with rFVIIa to those treated with placebo. The planned enrollment size is 110 patients in Canada. ${ }^{25}$

\section{Anticoagulant-Related Intracerebral Hemorrhage}

About one in five cases of ICH are anticoagulant related. ${ }^{26}$ Warfarin remains the most commonly used oral anticoagulant, and interventions that reverse anticoagulation due to warfarin are crucial. Options for reversal of anticoagulation due to warfarin include fresh frozen plasma (FFP), vitamin K, prothrombin complex concentrates (PCCs), and rfVIIa, alone or in combination. ${ }^{27}$

Fresh frozen plasma and vitamin $\mathrm{K}$ remain the most commonly used reversal agents. Delays in administration of FFP have been reported and every 30-minute delay in administration of FFP is associated with a $20 \%$ reduction in successful INR correction by 24 hours after presentation. ${ }^{28}$ Vitamin $K$ should be administered to all patients with warfarin-related $\mathrm{ICH}$, but it is not sufficiently effective in a short period to suffice as the only treatment for ICH. Prothrombin complex concentrates may be classified as three factor (sufficient levels of factors II, IX, and X, and low levels of factor VII) or four factor (sufficient levels of factors II, VII, IX, and X, as well as proteins $\mathrm{C}$ and $\mathrm{S}$ ). Prothrombin complex concentrates are more effective than FFP in rapidly reversing anticoagulation due to warfarin, ${ }^{29}$ require less volume infusion, and can be delivered rapidly. As such, in addition to vitamin $\mathrm{K}$ administration, four-factor PCCs is recommended over FFP for patients with major bleeding. ${ }^{27}$ Four-factor PCCs had been unavailable in the United States until the U.S. Food and Drug Administration (FDA) approved Kcentra (CSL Behring GmbH, Marburg, Germany) in May 2013. Given the lifethreatening consequence of delays in reversal of anticoagulation in patients with warfarin-related ICH, we believe fourfactor PCCs should be first-line therapy in addition to administration of vitamin $\mathrm{K}$ and additional FFP as needed.

Novel anticoagulant agents including direct thrombin inhibitors such as dabigatran and factor Xa inhibitors such as rivaroxaban and apixaban have recently been approved for treatment of atrial fibrillation and venous thromboembolism. ${ }^{30-33}$ Although case vignettes of ICH and other major bleeding associated with these agents have been published, no proven reversal regimens exist. As such, options for treating ICH related to these novel anticoagulants are FFP, PPCs, and rFVIIa.

\section{Surgical Management}

Surgical management of ICH should be discussed in terms of approach (craniotomy/craniectomy vs. minimally invasive approaches) and ICH location (supra- or infratentorial location). Emergent craniectomy may be life-saving in patients with posterior fossa $\mathrm{ICH}$, and surgery is recommended in patients with posterior fossa hemorrhages larger than $3 \mathrm{~cm}$ in diameter, brainstem compression, or hydrocephalus from ventricular obstruction. ${ }^{1}$ Because patients with posterior fossa lesions may regain close to full function with this life- 
saving surgery, there is little controversy with this recommendation and treatment approach. In the context of the STICH II results discussed below, it remains unclear whether craniotomy/craniectomy is beneficial in supratentorial ICH.

Minimally invasive hematoma evacuation after ICH offers the benefit of removal of offending factors in blood that may cause secondary injury without the morbidity associated with craniotomy. The largest trial published to date randomized 377 patients with small basal ganglia ICH $(25-40 \mathrm{~mL})$ to craniopuncture followed by urokinase and clot aspiration versus medical therapy alone. ${ }^{34}$ At 90 days, there was no significant difference in mortality (6.7\% vs. $8.8 \%, p=0.44)$, and the medical therapy group was more likely to be dependent (modified Rankin score $3-6)$ (63\% vs. 41\%, $p<0.01$ ). ${ }^{34}$ The MISTIE III and CLEAR III trials will confirm whether this approach is associated with improved long-term outcomes in treated patients.

\section{Neuroprotective Strategies}

In addition to direct tissue destruction and mass effect on adjacent tissues, ICH may trigger many deleterious inflammatory cascades. These include tissue necrosis, cellular apoptosis, and edema. Various neuroprotective approaches are being evaluated for efficacy in reducing secondary injury after ICH. To our knowledge, none are currently mature enough for phase III clinical trial evaluation.

\section{Phase III Clinical Trials in Intracerebral Hemorrhage}

\section{Clinical Trials Published in 2013}

Two critical phase III clinical trials of ICH were published in the past year: (1) rapid blood-pressure lowering in patients with acute intracerebral hemorrhage (INTERACT2) $)^{35}$ and (2) early surgery versus initial conservative treatment in patients with spontaneous supratentorial lobar intracerebral hematomas (STICH II), a randomized trial. ${ }^{36}$

INTERACT2 randomized 2839 patients with spontaneous ICH diagnosed on CT or MRI who presented within 6 hours to an intensive treatment to lower blood pressure (target systolic of $<140 \mathrm{~mm} \mathrm{Hg}$ within 1 hour) or current guidelinerecommended treatment (target systolic level of $<180 \mathrm{~mm}$ $\mathrm{Hg}$ ). Patients were eligible if they had a systolic blood pressure (SBP) between 150 and $220 \mathrm{~mm} \mathrm{Hg}$ and had no contraindication to blood pressure lowering. Antihypertensive medication choices were at the discretion of the treating physician. Of 2,794 patients for whom the primary outcome of death or major disability (defined as a score of 3-6 on the modified Rankin scale) was available at 90 days, $52 \%$ in the intensive group versus $56 \%$ in the standard treatment group had the primary outcome (odds ratio $=0.87 ; 95 \% \mathrm{CI}=0.75-1.01$, $p=0.06$ ). The authors concluded that intensive blood pressure reduction in acute ICH was safe, but did not significantly reduce death or major disability. ${ }^{35}$

The STICH II trial hypothesized that early surgery could improve outcome in conscious patients in whom there was a superficial ICH of 10 to $100 \mathrm{~mL}$ and no evidence of IVH. STICH II randomized 601 patients from 78 centers in 27 countries to early surgery (within 12 hours of ictus) or initial conservative treatment. The primary outcome was a prognosis-based favorable or unfavorable outcome based on the Extended Glasgow Outcome Scale (GOSE) at 6 months after randomization. Forty-one percent (123 out of 297) of patients in the early surgery group had a favorable outcome at 6 months compared with $38 \%$ (108 out of 286) of patients in the initial conservative treatment group ( $\mathrm{OR}=0.86,95 \% \mathrm{CI}=0.62-$ $1.20, p=0.367)$. Notably, $21 \%$ of the initial conservative treatment group in STICH II had surgery, thereby potentially blunting any treatment effect that may have been observed. Ultimately, it remains unclear what subgroup of ICH patients would benefit from surgery although ongoing studies may better define this. ${ }^{36}$

\section{Ongoing Phase III ICH Clinical Trials}

To our knowledge, there are three ongoing phase III acute interventional trials in $\mathrm{ICH}$. The Antihypertensive Treatment of Cerebral Hemorrhage (ATACH) 2 trial is randomizing ICH patients to a goal SBP of $<140 \mathrm{~mm} \mathrm{Hg}$ versus $<180 \mathrm{~mm} \mathrm{Hg}$ within 3.5 hours of symptom onset. Blood pressure targets are to be maintained for 24 hours after randomization. Both arms of the trial receive nicardipine in standard dosing regimens established per trial protocol. ${ }^{37}$ Labetalol may also be used if maximum amounts of nicardipine are used. Given the relatively delayed onset to initiation of therapy ( $<6$ hours), the multiple medications used and the difference in time from randomization to initiation of antihypertensive therapy between the treatment groups in INTERACT2, ${ }^{35}$ ATACH 2 should provide further data on the safety and efficacy of ultraearly (< 3.5 hours) rapid reduction of blood pressure using a single antihypertensive agent after ICH.

With a planned enrollment of $500 \mathrm{ICH}$ patients with IVH, the Clot Lysis: Evaluating Accelerated Resolution of Intraventricular Hemorrhage (CLEAR) III trial is randomizing ICH patients who require an EVD for obstructive hydrocephalus due to third or fourth ventricular blood to recombinant tissue plasminogen activator (rt-PA) versus placebo for resolving the IVH. Treatment must begin within 72 hours of the diagnostic CT scan. In addition to third or fourth ventricular obstruction, eligible patients must have $<30 \mathrm{~mL}$ of $\mathrm{ICH}$. The primary outcome measure is the modified Rankin scale score at 180 days. $^{38}$

The Minimally Invasive Surgery Plus Rt-PA for ICH Evacuation (MISTIE) Phase III trial is a randomized clinical trial of minimally invasive surgery plus rt-PA versus medical therapy alone after ICH. Eligible patients must have supratentorial $\mathrm{ICH} \geq 30 \mathrm{~mL}$, symptom onset within 24 hours of diagnostic CT and initiation of treatment from 12 to 72 hours of the diagnostic $\mathrm{CT}$, with the first dose given within 76 hours of the diagnostic CT. The primary efficacy outcome measure is a $12 \%$ increase in the proportion of rt-PA treated patients with a modified Rankin score of 0 to 3 compared with medically treated patients at 180 days. The primary safety outcome measure is the rate of mortality, rebleeding, and infection at 30 days. $^{39}$

These ongoing trials complement the recently completed trials and have a direct clinical impact on the bedside 
Table 1 Recently completed and ongoing phase III clinical trials of ICH

\begin{tabular}{|c|c|c|c|}
\hline Trial & $\begin{array}{l}\text { Number of } \\
\text { trial participants }\end{array}$ & Intervention & Outcomes in patients with $\mathrm{ICH}$ \\
\hline $\begin{array}{l}\text { INTERACT2 } \\
\text { Anderson et al }(2013)^{35}\end{array}$ & 2,839 & $\begin{array}{l}\text { Blood pressure reduction } \\
\text { to goal SBP }<140 \mathrm{~mm} \mathrm{Hg} \\
\text { within } 6 \mathrm{~h} \text { of onset }\end{array}$ & $\begin{array}{l}\text { Found to be safe. Did not reduce severe } \\
\text { disability or death }\end{array}$ \\
\hline $\begin{array}{l}\text { STICH II } \\
\text { Mendelow et al }(2013)^{36}\end{array}$ & 601 & $\begin{array}{l}\text { Early surgery vs. } \\
\text { conservative treatment }\end{array}$ & No clear benefit from early surgery \\
\hline $\begin{array}{l}\text { MISTIE III } \\
\text { Hanley et al }\end{array}$ & Projected 500 & $\begin{array}{l}\text { Minimally invasive } \\
\text { surgery plus rt-PA }\end{array}$ & Currently enrolling \\
\hline $\begin{array}{l}\text { CLEAR III } \\
\text { Ziai et al }(2013)^{38}\end{array}$ & Projected 500 & $\begin{array}{l}\text { rt-PA in patients who } \\
\text { require EVD for obstructive } \\
\text { hydrocephalus due to } \\
3^{\text {rd }} \text { or } 4^{\text {th }} \text { ventricular blood }\end{array}$ & Currently enrolling \\
\hline $\begin{array}{l}\text { ATACH2 } \\
\text { Qureshi et al }(2011)^{37}\end{array}$ & Projected 1280 & $\begin{array}{l}\text { Blood pressure reduction } \\
\text { to goal SBP }<140 \mathrm{~mm} \mathrm{Hg} \\
\text { within } 3.5 \mathrm{~h} \text { of onset }\end{array}$ & Currently enrolling \\
\hline
\end{tabular}

Abbreviations: EVD, extraventricular drain; ICH, intracerebral hemorrhage; rt-PA, recombinant tissue plasminogen activator; SBP = systolic blood pressure.

management of ICH. - Table 1 summarizes recently completed and ongoing phase III clinical trials of ICH.

\section{Conclusions}

Intracerebral hemorrhage remains a challenging clinical and public health problem. Fortunately, recently completed and ongoing acute clinical trials have and will meaningfully impact its clinical management. Although no trials to date have shown a definite improvement in functional outcomes with a given intervention, recent trials have informed blood pressure management and the need for surgery after acute ICH. Ongoing trials should add to current knowledge and are promising for establishing the efficacy of the medical and surgical interventions being tested.

\section{Acknowledgments}

The authors wish to thank Dr. Achala Vagal for assistance with the images.

\section{References}

1 Morgenstern LB, Hemphill JC III, Anderson C, et al; American Heart Association Stroke Council and Council on Cardiovascular Nursing. Guidelines for the management of spontaneous intracerebral hemorrhage: a guideline for healthcare professionals from the American Heart Association/American Stroke Association. Stroke 2010;41(9):2108-2129

2 Roger VL, Go AS, Lloyd-Jones DM, et al. Heart disease and stroke statistics-2012 update: a report from the American Heart Association. Circulation 2012;125(1):e2-e220

3 Toyoda K, Steiner T, Epple C, et al. Comparison of the European and Japanese guidelines for the acute management of intracerebral hemorrhage. Cerebrovasc Dis 2013;35(5):419-429
4 Brott T, Broderick J, Kothari R, et al. Early hemorrhage growth in patients with intracerebral hemorrhage. Stroke 1997;28(1):1-5

5 Broderick JP, Diringer MN, Hill MD, et al; Recombinant Activated Factor VII Intracerebral Hemorrhage Trial Investigators. Determinants of intracerebral hemorrhage growth: an exploratory analysis. Stroke 2007;38(3):1072-1075

6 Davis SM, Broderick J, Hennerici M, et al; Recombinant Activated Factor VII Intracerebral Hemorrhage Trial Investigators. Hematoma growth is a determinant of mortality and poor outcome after intracerebral hemorrhage. Neurology 2006;66(8):1175-1181

7 Mayer SA, Brun NC, Begtrup K, et al; Recombinant Activated Factor VII Intracerebral Hemorrhage Trial Investigators. Recombinant activated factor VII for acute intracerebral hemorrhage. N Engl J Med 2005;352(8):777-785

8 Mayer SA, Brun NC, Begtrup K, et al; FAST Trial Investigators. Efficacy and safety of recombinant activated factor VII for acute intracerebral hemorrhage. N Engl J Med 2008;358(20):2127-2137

9 Hemphill JC III, Farrant M, Neill TA Jr. Prospective validation of the ICH Score for 12-month functional outcome. Neurology 2009; 73(14):1088-1094

10 Hemphill JC III, Bonovich DC, Besmertis L, Manley GT, Johnston SC. The ICH score: a simple, reliable grading scale for intracerebral hemorrhage. Stroke 2001;32(4):891-897

11 Morgan T, Awad I, Keyl P, Lane K, Hanley D. Preliminary report of the clot lysis evaluating accelerated resolution of intraventricular hemorrhage (CLEAR-IVH) clinical trial. Acta Neurochir Suppl (Wien) 2008;105:217-220

12 Naff NJ, Carhuapoma JR, Williams MA, et al. Treatment of intraventricular hemorrhage with urokinase: effects on 30-day survival. Stroke 2000;31(4):841-847

13 Rost NS, Smith EE, Chang Y, et al. Prediction of functional outcome in patients with primary intracerebral hemorrhage: the FUNC score. Stroke 2008;39(8):2304-2309

14 Becker KJ, Baxter AB, Cohen WA, et al. Withdrawal of support in intracerebral hemorrhage may lead to self-fulfilling prophecies. Neurology 2001;56(6):766-772

15 Hanley DF, Awad IA, Vespa PM, Martin NA, Zuccarello M. Hemorrhagic stroke: introduction. Stroke 2013;44(6, Suppl 1):S65-S66

16 Goldstein JN, Fazen LE, Snider R, et al. Contrast extravasation on CT angiography predicts hematoma expansion in intracerebral hemorrhage. Neurology 2007;68(12):889-894 
17 Wada R, Aviv RI, Fox AJ, et al. CT angiography “spot sign” predicts hematoma expansion in acute intracerebral hemorrhage. Stroke 2007;38(4):1257-1262

18 Demchuk AM, Dowlatshahi D, Rodriguez-Luna D, et al; PREDICT/ Sunnybrook ICH CTA study group. Prediction of haematoma growth and outcome in patients with intracerebral haemorrhage using the CT-angiography spot sign (PREDICT): a prospective observational study. Lancet Neurol 2012;11(4):307-314

19 Steiner T, Kaste M, Forsting M, et al; The European Stroke Initiative Writing Committee and the Writing Committee for the EUSI Executive Committee. Recommendations for the management of intracranial haemorrhage - part I: spontaneous intracerebral haemorrhage. Cerebrovasc Dis 2006;22(4):294-316

20 Broderick JP, Adams HP Jr, Barsan W, et al. Guidelines for the management of spontaneous intracerebral hemorrhage: astatement for healthcare professionals from a special writing group of the Stroke Council, American Heart Association. Stroke 1999;30(4):905-915

21 Broderick J, Connolly S, Feldmann E, et al; American Heart Association/American Stroke Association Stroke Council; American Heart Association/American Stroke Association High Blood Pressure Research Council; Quality of Care and Outcomes in Research Interdisciplinary Working Group. Guidelines for the management of spontaneous intracerebral hemorrhage in adults: 2007 update: a guideline from the American Heart Association/American Stroke Association Stroke Council, High Blood Pressure Research Council, and the Quality of Care and Outcomes in Research Interdisciplinary Working Group. Circulation 2007;116(16):e391-e413

22 Anderson CS, Huang Y, Wang JG, et al; INTERACT Investigators. Intensive blood pressure reduction in acute cerebral haemorrhage trial (INTERACT): a randomised pilot trial. Lancet Neurol 2008; 7(5):391-399

23 Diringer MN, Skolnick BE, Mayer SA, et al. Thromboembolic events with recombinant activated factor VII in spontaneous intracerebral hemorrhage: results from the Factor Seven for Acute Hemorrhagic Stroke (FAST) trial. Stroke 2010;41(1):48-53

24 Mayer SA, Davis SM, Skolnick BE, et al; FAST trial investigators. Can a subset of intracerebral hemorrhage patients benefit from hemostatic therapy with recombinant activated factor VII? Stroke 2009; 40(3):833-840

25 The Spot Sign for Predicting \& Treating ICH Growth (STOP-IT); The Spot Sign Selection of Intracerebral Hemorrhage to Guide Hemostatic Therapy (SPOTLIGHT). 2013. http://www.clinicaltrials.gov. Accessed September 30, 2013

26 Flaherty ML, Kissela B, Woo D, et al. The increasing incidence of anticoagulant-associated intracerebral hemorrhage. Neurology 2007;68(2):116-121

27 Holbrook A, Schulman S, Witt DM, et al. Evidence-based management of anticoagulant therapy: Antithrombotic Therapy and
Prevention of Thrombosis, 9th ed: American College of Chest Physicians Evidence-Based Clinical Practice Guidelines. Chest 2012;141(2 Suppl):e152S-184S

28 Goldstein JN, Thomas SH, Frontiero V, et al. Timing of fresh frozen plasma administration and rapid correction of coagulopathy in warfarin-related intracerebral hemorrhage. Stroke 2006;37(1): 151-155

29 Sarode R, Milling TJ Jr, Refaai MA, et al. Efficacy and safety of a 4-factor prothrombin complex concentrate in patients on vitamin K antagonists presenting with major bleeding: a randomized, plasma-controlled, phase IIIb study. Circulation 2013; 128(11):1234-1243

30 Granger CB, Alexander JH, McMurray JJ, et al; ARISTOTLE Committees and Investigators. Apixaban versus warfarin in patients with atrial fibrillation. N Engl J Med 2011;365(11):981-992

31 Connolly SJ, Ezekowitz MD, Yusuf S, et al; RE-LY Steering Committee and Investigators. Dabigatran versus warfarin in patients with atrial fibrillation. N Engl J Med 2009;361(12): 1139-1151

32 Büller HR, Prins MH, Lensin AW, et al; EINSTEIN-PE Investigators. Oral rivaroxaban for the treatment of symptomatic pulmonary embolism. N Engl J Med 2012;366(14):1287-1297

33 Patel MR, Mahaffey KW, Garg J, et al; ROCKET AF Investigators. Rivaroxaban versus warfarin in nonvalvular atrial fibrillation. $\mathrm{N}$ Engl J Med 2011;365(10):883-891

34 Wang WZ, Jiang B, Liu HM, et al. Minimally invasive craniopuncture therapy vs. conservative treatment for spontaneous intracerebral hemorrhage: results from a randomized clinical trial in China. Int J Stroke 2009;4(1):11-16

35 Anderson CS, Heeley E, Huang Y, et al; INTERACT2 Investigators. Rapid blood-pressure lowering in patients with acute intracerebral hemorrhage. N Engl J Med 2013;368(25):2355-2365

36 Mendelow AD, Gregson BA, Rowan EN, Murray GD, Gholkar A, Mitchell PM; STICH II Investigators. Early surgery versus initial conservative treatment in patients with spontaneous supratentorial lobar intracerebral haematomas (STICH II): a randomised trial. Lancet 2013;382(9890):397-408

37 Qureshi AI, Palesch YY. Antihypertensive Treatment of Acute Cerebral Hemorrhage (ATACH) II: design, methods, and rationale. Neurocrit Care 2011;15(3):559-576

38 Ziai WC, Tuhrim S, Lane K, et al; CLEAR III Investigators. A multicenter, randomized, double-blinded, placebo-controlled phase III study of Clot Lysis Evaluation of Accelerated Resolution of Intraventricular Hemorrhage (CLEAR III). Int J Stroke 2013

39 Hanley DF, et al. Minimally Invasive Surgery Plus Rt-PA for ICH Evacuation Phase III (MISTIE III). 2013. http://www.clinicaltrials. gov. Accessed September 30, 2013 